\title{
FLUTUAÇÃO POPULACIONAL E DANOS DE Grapholita molesta (LEPIDOPTERA: TORTRICIDAE) EM DOIS SISTEMAS DE PRODUÇÃO DE PESSEGUEIROS ${ }^{1}$
}

\author{
ALEX SANDRO POLTRONIERI ${ }^{2}$,LINO BITTENCOURT MONTEIRO ${ }^{3}$, LOUISE LARISSA MAY-DE-MIO ${ }^{4}$
}

RESUMO - Com o objetivo de conhecer a flutuação populacional de Grapholita molesta e verificar a fase fenológica do pessegueiro mais sensível ao ataque do inseto, em dois sistemas de produção, selecionaram-se quatro pomares de pessegueiro, sendo dois conduzidos no sistema de produção convencional (PC) e dois no sistema de boas práticas agrícolas (BPA), durante as safras de 2005/ 06 e 2006/07, em Araucária-PR. Para verificar a flutuação, foram instaladas duas armadilhas modelo Delta por pomar, iscadas com feromônio sexual sintético, onde se registravam semanalmente as capturas dos adultos de G. molesta. Para determinar a fase mais sensível do pessegueiro ao ataque de G. molesta, foram avaliados as brotações e os frutos do ramo primário voltado para o poente, em vinte plantas por pomar, nas fases de raleio, endurecimento do caroço e maturação. A população de G. molesta, na safra de 2005/06, foi superior à de 2006/07, com os pomares BPA apresentando ocorrências superiores aos PCs. As maiores populações do inseto ocorreram após a colheita. A fase de maturação foi a mais suscetível em ambos os sistemas de produção, com os pomares BPA apresentando menor percentual de danos.

Termos para Indexação: Mariposa-oriental, pessegueiro, danos.

\section{POPULATION FLUCTUATION AND DAMAGE FROM Grapholita molesta (LEPIDOPTERA: TORTRICIDAE) IN TWO PEACH PRODUCTION SYSTEMS}

\begin{abstract}
With the objective to know the population fluctuation of Grapholita molesta and to verify the phenological stage that the peach tree is more sensitive to the attack of the insect in two production systems, four peach orchards were selected, two under the conventional production system (CP) and two under the good agricultural practice system (GAP), in 2005/06 and 2006/07 growing seasons, in Araucária, PR, Brazil. To verify the fluctuation, two Delta model traps were installed per orchard, baited with synthetic sexual pheromone, where the capture of G. molesta adults was recorded weekly. To determine the most sensitive phase of the peach tree to G. molesta attack, the bud and fruits were evaluated on the primary branch turned to the West on twenty plants per orchard, in the thinning, stone hardening and maturing phases. The G. molesta population in the 2005/2006 growing season was greater than in 2006/07, and the GAP orchards presented greater occurrences than the CP. The biggest populations of the insect occurred postharvest. The maturation phase was the most susceptible in both production systems, but there was a lower percentage of damage to the GAP orchards.
\end{abstract}

Index Terms: Oriental fruit moth, peach tree, damages.

\section{INTRODUÇÃO}

A intensidade dos impactos ambientais causados pelas práticas agrícolas está diretamente relacionada com o sistema de produção utilizado (Fadini \& Louzada, 2001). Na tentativa de uniformizar as atividades em pomares de pessegueiro, foram elaboradas as Normas da Produção Integrada (Fachinello et al., 2003), as quais orientam, por exemplo, o manejo de pragas.

A mariposa oriental, Grapholita molesta (Busck, 1916) (Lepidoptera: Tortricidae), é uma das principais pragas do pessegueiro (Monteiro \& Hickel, 2004), danificando brotações e frutos (Salles, 1991). Em brotações, o inseto destrói o meristema apical (Gonzalez, 2003) e, nos frutos, abre galerias na polpa, inviabilizando o pêssego para o consumo (Salles, 1991).

Segundo Salles (2000), a preferência alimentar de $G$. molesta é por frutos na fase de amadurecimento, em detrimento a verdes ou em ponto de colheita; assim, o manejo do inseto, em algumas cultivar, pode ser feito considerando as fases fisiológicas do fruto. Os frutos passam por duas fases fisiológicas no período em que estão no campo: crescimento e maturação. Na primeira fase, ocorre a multiplicação celular, iniciando na fecundação do ovário ao endurecimento do caroço. A segunda fase é caracterizada pela expansão e enchimento celular (Chitarra \& Chitarra, 2005). Os produtores, para minimizar os danos, iniciam as pulverizações após a floração até próximo à colheita, afetando toda a entomofauna, contaminando o ambiente e colocando em risco a saúde humana (Botton et al., 2001)

O monitoramento de pragas pode fornecer subsídios para decisões quanto às pulverizações, sendo recomendado o controle químico quando as populações de pragas atingem níveis de controle (Carvalho, 1990). O monitoramento de G. molesta é

${ }^{1}$ (Trabalho 149-07). Recebido em: 15-06-2007. Aceito para publicação em: 06-06-2007.

${ }^{2} \mathrm{Eng}^{\circ}$ agr $^{\circ}$, aluno de mestrado do Programa de Pós-Graduação em Agronomia, Dept. de Fitotécnia, CCA/UFPR, Curitiba- PR. Bolsista Capes alex.poltronieri@yahoo.com.br

${ }^{3} \mathrm{Eng}^{\circ}$ agr $^{\circ}$, Dr. Professor do Programa de Pós-Graduação em Agronomia, Dept. de Fitotécnia, CCA/UFPR, Curitiba-PR. lbmonteiro@terra.com.br ${ }^{4}$ Eng $^{\mathrm{a}}$ agr $^{\mathrm{a}}$, Dra. Professora do Programa de Pós-Graduação em Agronomia, Dept. de Fitotécnia, CCA/UFPR, Curitiba-PR.

Rev. Bras. Frutic., Jaboticabal - SP, v. 30, n. 3, p. 628-633, Setembro 2008 
realizado por meio de feromônio sexual sintético (Arioli et al., 2005), sendo o nível de controle recomendado em 20 insetos/ armadilha/semana (Salles, 1991). Entretanto, o monitoramento pode ser complementado pela agregação de conhecimentos sobre as fases onde o pessegueiro é mais suscetível ao ataque de $G$. molesta.

Este trabalho teve como objetivos determinar a flutuação populacional e danos de G. molesta em dois sistemas de produção do pessegueiro e verificar a fase fenológica do pessegueiro mais suscetível ao ataque do inseto em pomares Araucária-PR.

\section{MATERIAL E MÉTODOS}

\section{Descrição da área experimental}

Os experimentos foram conduzidos em quatro pomares de pessegueiros da variedade 'Chimarrita', com onze anos de idade, plantadas no espaçamento $4 \times 6 \mathrm{~m}$ e com área média de 0,36 hectares, correspondendo ao tamanho médio dos pomares do município de Araucária-PR (Lat: 253'' S; Lon: 49²4' W e 897 m de altitude). As áreas distanciavam-se cerca de 10 mil metros entre si, e conforme o manejo adotado pelos produtores, os pomares foram divididos em dois grupos:

A) Grupo Boas Práticas Agrícolas (BPA): formado por dois pomares que apresentavam como característica comum a execução de Boas Práticas Agrícolas (BPA) e processo de adoção da Produção Integrada de Frutas (PIF) a partir de 2002, de acordo com as normas técnicas e documentos de acompanhamento da produção integrada de pêssego (Fachinello et al., 2003).

B) Grupo Convencional (PC): formado por dois pomares conduzidos sem normas específicas, baseando-se na experiência do produtor, prevalecendo os tratamentos fitossanitários realizados de acordo com calendário, sem monitoramento de pragas e doenças, manejo de ervas e práticas conservacionistas de solo.

Os produtos fitossanitários utilizados pelos produtores, para o manejo de pragas nos quatro pomares de estudo, encontram-se na Tabela 1.

Flutuação populacional de machos adultos de $G$. molesta A população de adultos de G. molesta foi monitorada entre julho de 2005 e março de 2006 e entre maio e novembro de 2006, por meio de armadilhas Delta, contendo um difusor com feromônio sexual sintético (Biografolita ${ }^{\circledR}$, São Paulo), sendo instaladas duas armadilhas em cada pomar, localizadas em bordas opostas do pomar. As armadilhas foram instaladas na parte externa das plantas a 1,70 m de altura, no ramo primário voltado para o poente (Salles \& Marini, 1989). Os difusores de feromônio foram substituídos a cada seis semanas e o piso adesivo trocado quando houve necessidade. As avaliações das armadilhas foram realizadas semanalmente, e os insetos capturados foram quantificados e retirados. O nível de controle (NC) adotado foi de 20 indivíduos capturados/armadilha/semana (Salles, 1991).

A análise estatística para verificar a relação entre capturassemanais e dados climáticos foi realizada por meio do Teste de correlação de Pearson. Os dados climáticos da região de Araucária foram fornecidos pelo SIMEPAR (Instituto Tecnológico Sistema de Informações Meteorológicas do Paraná).

\section{Avaliação de danos em brotações e frutos}

A avaliação de danos em brotações foi realizada em vinte pessegueiros por pomar. Em cada planta, foi escolhido o ramo primário, voltado para o poente, onde se contaram todos os frutos e brotações. As avaliações foram feitas em duas fases fisiológicas do fruto: a) crescimento, subdividida em raleio (R) e endurecimento do caroço (EC) (verificado pela abertura do fruto e constatação do seu endurecimento, não se partindo , quando forçado); b) maturação (M).

Consideraram-se brotações atacadas os ramos do ano com o meristema apical destruído, com presença de galerias ou exsudação de goma, verificado durante avaliação visual, sendo estes identificados com fita, para não serem incluídos em leituras posteriores (Salles \& Marini, 1989). As avaliações de brotações foram feitas em $1^{\circ}$-10-05 e 22-08-06 (raleio); em 25-10-05 e 14-10-06 (endurecimento do caroço); e em 17-12-05 e 25-11-06 (colheita).

As avaliações em frutos foram feitas nas datas em que se realizou a avaliação nas brotações. Na fase de raleio, retiraram-se os frutos danificados e posteriormente procedeu-se ao raleio; na fase de endurecimento do caroço, retiraram-se apenas os frutos atacados; na colheita, realizada entre 28-11 e 17-12-05 e entre 0311 e 25-11-06, todos os frutos foram coletados e avaliados.

O percentual médio de brotações e frutos danificados durante as fases avaliadas foi submetido à análise de variância, sendo as médias comparadas pelo teste de Duncan $(\mathrm{p}>0,05)$.

\section{RESULTADOS E DISCUSSÃO}

Flutuação populacional de machos adultos de G. molesta As capturas de $G$. molesta, em 2005/06, foram superiores às registradas na safra 2006/07 (Figura 1).

A população de G. molesta, em 2005/06, foi superior no pomar BPA2 quando comparado ao BPA1 (Figura 1). Os dois primeiros picos acima do NC, registrados no BPA2, ocorreram na fase anterior ao raleio, entretanto o controle não foi realizado, pois os pessegueiros estavam em florescimento, portanto não sofrendo ameaças de ataque. Os dois picos registrados podem ser atribuídos aos insetos que passaram o inverno em diapausa (Arioli et al., 2005), pois a mariposa oriental apresenta uma diapausa não-sincronizada com a temperatura (Hickel, 2003), com populações emergindo em períodos onde há baixa disponibilidade alimentar. O terceiro pico populacional (14-10-05) pode ter origem das populações que ocorreram na floração, pois quando se calculam os graus-dia, pela metodologia usada por Grellmann et al. (1992), verificou-se o acúmulo de 493,2 graus-dia entre os picos observados em 19-8 e 14-10, o que permite o desenvolvimento de uma geração de G. molesta. Essa população de adultos ultrapassou o NC, portanto com possibilidade de causar danos na fase de maturação (25-10-05), que é a fase mais suscetível (Salles, 2000); por esse motivo, foi realizada uma pulverização em 21-10-05 (Tabela 1). No BPA1, as capturas foram inferiores a nove indivíduos por armadilha até a colheita, e as três pulverizações realizadas, embora afetem a população de G. molesta (Gonring et al., 1999), objetivaram o controle de mosca-das-frutas (Tabela 1). Nos pomares PC, na safra de 2005/06, as capturas de $G$. 
molesta ficaram abaixo do NC até a colheita (Figura 1), provavelmente devido às pulverizações realizadas de forma seqüencial (Tabela 1). Nestes pomares, observou-se o aumento populacional de G. molesta após a colheita, devido à interação de fatores, tais como, ausências de pulverizações, oferta alimentar (brotações) e fatores abióticos (temperatura). Na safra de 2005/ 06, o teste de Correlação de Pearson mostrou relações entre as capturas do inseto nos pomares avaliados e as temperaturas máximas $(\mathrm{r}=0,72)$ e médias $(\mathrm{r}=0,69)$, estando estes resultados de acordo com os obtidos por Arioli et al. (2005).

Na segunda safra (2006/07), as populações de G. molesta não atingiram o NC nos pomares BPA e PC até a colheita (Figura 2). As baixas populações podem ter ocorrido em função da baixa precipitação que houve nesta safra, pois, entre abril e outubro de 2006, a precipitação mensal foi, em média, de $43 \mathrm{~mm}$, ou seja, $66 \%$ inferior à ocorrida no mesmo período no ano anterior $(128 \mathrm{~mm})$. Cividanes \& Martins (2006) relatam que a baixa umidade pode ter sido um dos fatores que contribuíram para a ocorrência de baixas populações de G. molesta em pomares de pessegueiro de São Paulo. Löeck et al. (1991) atribuíram à estiagem as baixas populações de G. molesta em Pelotas, na safra 1988/89. e frutos

Avaliação de danos de Grapholita molesta em brotações

Os danos de G. molesta em frutos tiveram diferentes intensidades nas fases de raleio (FR), endurecimento do caroço (FEC) e maturação (FM), sendo a última fase a mais atacada pelo inseto em ambas as safras (Tabela 2).

Na safra de 2005/06, os danos em frutos na FR ( $\left.1^{\circ}-10-05\right)$ foram baixos em todos os pomares, sendo os danos maiores no PC1 (Tabela 2), embora as capturas de G. molesta tenham sido baixas e o pomar tenha recebido três pulverizações nessa fase (Tabela 1). No BPA2, não ocorreram danos nos frutos, mesmo que as capturas tenham sido superiores ao $\mathrm{NC}$ na fase de floração e sem o controle químico. Esse fato sugere que as lagartas, oriundas das populações na fase de floração, não estão diretamente relacionadas com os danos na FR, como constatou Gonzalez (2003) no Chile. Nessa fase, as temperaturas médias de Araucária estão abaixo de $15^{\circ} \mathrm{C}$, o que influencia na eclosão e no comportamento de lagartas (Grellmann et al., 1992).

$\mathrm{Na}$ FEC, os danos nos pomares BPA não apresentaram diferenças (Tabela 2), mesmo que, no BPA2, tenham ocorrido dois e um picos populacionais acima do $\mathrm{NC}$, respectivamente, na fase de floração e na FEC. Isso sugere que a população de lagartas originada na floração não se tenha desenvolvido suficientemente para danificar os frutos na FEC e/ou as condições ambientais não contribuíram para isso, embora o acúmulo de graus-dia entre os dois picos seja suficiente para produzir uma geração. A pulverização ocorrida nessa fase pode ter influência sobre os danos na fase seguinte (FM) (Tabela 1).

Nos pomares PCs, os danos foram superiores a $2 \%$, valores maiores do que os observados nos BPAs, mesmo com a realização de duas pulverizações em cada pomar (Tabela 1).Os inseticidas deveriam limitar os danos de lagartas em FEC (Figura 1), pois são registrados para a praga (Andrei, 1999; SEAB, 2008). Entretanto, isso não ocorreu, podendo ser devido a interações de fatores, tais como, efeito da temperatura e qualidade de pulverização.

Na FM, ocorreram os maiores níveis de danos em frutos, havendo diferenças entre os pomares (Tabela 2). O BPA1 teve o menor percentual de danos, sendo que a baixa população do inseto nesta fase (Figura 1) e a realização de 1 e 2 pulverizações para moscas-das-frutas (Tabela 1), respectivamente na FEC e FM, possam ter influenciado na redução do número de frutos danificados. A maior população de G. molesta verificada no BPA2, nas FEC e FM, pode ter contribuído para que os danos nesse pomar fossem $80,7 \%$ maiores do que os observados no BPA1.

Os PCs tiveram a maior incidência de danos em frutos na FM (Tabela 2), embora as capturas de G. molesta tenham sido inferiores às ocorridas no BPA(Figura 1), assim como maior número de inseticidas na FEC e FM (Tabela 1). O elevado percentual de danos ocorridos neste pomar sugere que as pulverizações para o controle de G. molesta foram ineficientes, fato que pode ser atribuído à ausência do monitoramento, que indicaria o momento adequado para a realização das pulverizações, e a escolha do inseticida fenthion, reconhecido como eficiente Anastrepha fraterculus (Diptera: Tephritidae) (Cruz et al., 2000).

Acumulando-se os danos das fases de FEC e FM, verificou-se que os pomares BPA foram menos atacados por $G$. molesta (Tabela 2). Botton et al. (2001) mostraram que cultivares de ciclo médio apresentam valores inferiores às cultivares tardias, devido às elevadas populações do inseto no período de maturação de frutos à colheita. Os resultados encontrados em Araucária, nos pomares BPA, foram contrários aos obtidos por Nunes et al. (2003) em São Jerônimo-RS, quando observaram maiores danos em pomares PIF em relação aos pomares de PC.

A diferença de danos entre os pomares BPA e PC pode estar relacionada com as diferenças de estratégias adotadas nos dois pomares, não somente no período de estudo, mas em função das adequações que foram realizadas nos BPAs entre os anos de 2002 e 2004, quando os produtores fizeram ajustes para se enquadrar nas normas da Boas Práticas Agrícolas e Produção Integrada de Frutas. Isto pode ser verificado pela melhor eficiência dos tratamentos realizados nos BPAs, caracterizado pela menor porcentagem de danos acumulados e menor número de pulverizações, o que deve proporcionar menor impacto ambiental.

$\mathrm{Na}$ safra de 2006/07, houve um período de baixas temperaturas e de geadas entre agosto e setembro que causaram a redução de frutos, em média de $50 \%$ nos pomares PC e de $84 \%$ no BPA1. O BPA2 não sofreu perdas de produção em função da pulverização de açúcar a 5\% sobre os pessegueiros, o que provocou a redução do ponto de congelamento. A população do inseto também foi inferior à registrada na safra anterior, não atingindo até a colheita o NC (Figura 2).

Pomares de ambos os sistemas não tiveram danos de frutos na FR, enquanto em FEC os pomares PC tiveram o maior percentual de frutos atacados (Tabela 2). A FM foi a fase mais suscetível ao ataque de G. molesta, embora os danos nesta safra tenham sido inferiores aos ocorridos na safra de 2005/06. Os danos no BPA1 foram elevados em função de o produtor optar em não realizar a colheita, pois os frutos sofreram lesões na epiderme causadas pelo frio, o que os inviabilizou à comercialização; houve 
apenas uma pulverização nesse pomar (Tabela 1).

Os danos em brotações foram baixos nas duas safras e não houve diferença significativa entre os dois sistemas de produção. Esses danos foram observados a partir da FEC e elevando-se durante a FM, mas sem ultrapassar 0,5\% de brotações atacadas (Tabela 2). Embora não tenham sido quantificados, verificou-se aumento dos danos em brotações após a colheita dos frutos, sendo este fato descrito por Martins $\& \operatorname{Link}(1978)$.
A FM foi caracterizada como a fase onde ocorreram os maiores danos em frutos e brotações, sendo a mais suscetível para a variedade 'Chimarrita' (Tabelas 2). Os danos nesta fase podem ser justificados pela preferência do inseto por frutos em maturação (Salles, 2000).

TABELA1 - Tratamentos fitossanitários realizados em quatro pomares conduzidos em dois sistemas de produção de pessegueiros 'Chimarrita'. Araucária-PR. 2005/06 e 2006/07.

\begin{tabular}{|c|c|c|c|c|c|}
\hline \multirow{2}{*}{ Data } & \multicolumn{2}{|r|}{$\mathrm{PC}^{2}{ }^{2}$} & \multirow{2}{*}{ Data } & \multicolumn{2}{|r|}{$\mathrm{PC}^{2}$} \\
\hline & Inseticida $^{1}$ & Praga-alvo & & Inseticida $^{1}$ & Praga-alvo \\
\hline $1^{\mathrm{o}}-09-05$ & fenthion & Grapholita molesta & $05-10-05$ & fenthion & Grapholita molesta \\
\hline $15-09-05$ & fenthion & Grapholita molesta & $27-10-05$ & fenthion & Grapholita molesta \\
\hline $30-09-05$ & fenthion & Grapholita molesta & $07-11-05$ & fenthion & Grapholita molesta \\
\hline $15-10-05$ & fenthion & Grapholita molesta & $23-11-05$ & dimethoate & Grapholita molesta \\
\hline $30-10-05$ & fenthion & Grapholita molesta & $11-12-05$ & dimethoate & Anastrepha sp. \\
\hline $15-11-05$ & fenthion & \multicolumn{4}{|l|}{ Grapholita molesta } \\
\hline 02-09-06 & fenthion & Anastrepha sp. & $15-09-06$ & fenthion & Anastrepha sp. e G. molesta \\
\hline $20-09-06$ & fenthion & Anastrepha sp. & $03-11-06$ & lambdacyhalothrin & Anastrepha sp. e G. molesta \\
\hline $10-10-06$ & fenthion & Anastrepha sp. & $17-11-06$ & deltamethrina & Anastrepha sp. e G. molesta \\
\hline \multicolumn{4}{|c|}{$\mathrm{BPA}^{3}$} & \multicolumn{2}{|r|}{$\mathrm{BPA} 2^{3}$} \\
\hline $15-10-05$ & fenthion & Anastrepha sp. & $21-10-05$ & fenitrothion & Grapholita molesta \\
\hline $08-11-05$ & fenthion & Anastrepha sp. & 04-11-05 & triflumurom & Grapholita molesta \\
\hline $29-11-05$ & dimethoate & Anastrepha sp. & $03-12-05$ & fenitrothion & Grapholita molesta \\
\hline \multirow{2}{*}{$20-10-06$} & \multirow{2}{*}{ fenthion } & \multirow{2}{*}{ Anastrepha sp. } & $10-10-06$ & dimethoate & Anastrepha sp. \\
\hline & & & $26-10-06$ & dimethoate & Anastrepha sp. \\
\hline
\end{tabular}

${ }^{1}$ Andrei, 1990; ${ }^{2}$ Produção Convencional (PC); ${ }^{3}$ Boas Praticas Agrícolas (BPA).

TABELA 2- Danos de Grapholita molesta em frutos e brotações de pessegueiro 'Chimarrita', conduzidos no sistema de Boas Práticas Agrícolas (BPA) e Produção Convencional (PC), nas fases de raleio ( R ), endurecimento do caroço (EC), Maturação (M) e danos acumulados. Araucária-PR. Safras 2005/06 e 2006/07.

\begin{tabular}{|c|c|c|c|c|c|c|c|c|c|c|c|c|c|c|c|}
\hline \multirow{3}{*}{ Safra } & \multirow{3}{*}{ Pomares } & \multicolumn{7}{|c|}{ Danos em frutos $(\%)$} & \multicolumn{7}{|c|}{ Danos em brotações (\%) } \\
\hline & & \multicolumn{2}{|c|}{$\mathrm{R}$} & \multicolumn{2}{|c|}{$\mathrm{EC}$} & \multicolumn{2}{|c|}{ M } & \multirow{2}{*}{$\mathrm{DA}^{1}$} & \multicolumn{2}{|c|}{$\mathrm{R}$} & \multicolumn{2}{|c|}{ EC } & \multicolumn{2}{|c|}{ M } & \multirow{2}{*}{$\mathrm{DA}^{2}$} \\
\hline & & $\mathrm{n}$ & Danos & $\mathrm{n}$ & Danos & $\mathrm{n}$ & Danos & & $\mathrm{n}$ & Danos & $\mathrm{n}$ & Danos & $\mathrm{n}$ & Danos & \\
\hline \multirow[t]{4}{*}{$2005 / 06$} & BPA1 & 4875 & $0,14 a$ & 2383 & $0,25 \mathrm{a}$ & 2377 & $0,63 a$ & $0,88 \mathrm{a}$ & 2602 & 0,00 & 2602 & $0,00 \mathrm{a}$ & 2602 & $0,15 \mathrm{a}$ & $0,15 \mathrm{a}$ \\
\hline & BPA2 & 2131 & $0,00 \mathrm{a}$ & 1508 & $0,93 a$ & 1494 & $3,27 \mathrm{~b}$ & $4,20 \mathrm{~b}$ & 2660 & 0,00 & 2660 & $0,00 \mathrm{a}$ & 2660 & $0,30 \mathrm{a}$ & $0,30 \mathrm{a}$ \\
\hline & PC1 & 7595 & $0,25 \mathrm{a}$ & 3386 & $2,21 b$ & 3311 & $3,32 b$ & $5,53 \mathrm{~b}$ & 2776 & 0,00 & 2776 & $0,03 a$ & 2776 & $0,10 \mathrm{a}$ & $0,13 \mathrm{a}$ \\
\hline & $\mathrm{PC} 2$ & 2208 & $0,00 \mathrm{a}$ & 2208 & $2,72 b$ & 2148 & $6,05 \mathrm{c}$ & $8,77 \mathrm{c}$ & 2125 & 0,00 & 2125 & $0,00 \mathrm{a}$ & 2125 & $0,37 \mathrm{a}$ & $0,37 \mathrm{a}$ \\
\hline \multirow[t]{4}{*}{$2006 / 07$} & BPA1 & 3 & 0,00 & 370 & 0,00 & 370 & $3,51 \mathrm{c}$ & 3,5 & 3317 & 0,00 & 3317 & $0,06 \mathrm{~b}$ & 3317 & $0,27 \mathrm{a}$ & $0,33 \mathrm{a}$ \\
\hline & BPA2 & 30483 & 0,00 & 5250 & $0,03 a$ & 5248 & $0,05 \mathrm{a}$ & $0,08 \mathrm{a}$ & 1650 & 0,00 & 1650 & $0,00 \mathrm{a}$ & 1650 & $0,00 \mathrm{a}$ & $0,00 \mathrm{a}$ \\
\hline & PC1 & 1883 & 0,00 & 1883 & $0,16 \mathrm{a}$ & 1880 & $0,85 b$ & $1,01 b$ & 4887 & 0,00 & 4887 & $0,00 \mathrm{a}$ & 4887 & $0,04 \mathrm{a}$ & $0,04 \mathrm{a}$ \\
\hline & PC2 & 1342 & 0,00 & 1342 & $0,22 a$ & 1339 & $1,34 b$ & $1,56 \mathrm{~b}$ & 3776 & 0,00 & 3776 & $0,00 \mathrm{a}$ & 3776 & $0,40 \mathrm{a}$ & $0,40 \mathrm{a}$ \\
\hline
\end{tabular}

Médias seguidas pela mesma letra não diferem estatisticamente, pelo teste de Duncan, ao nível de $5 \%$ de confiança.

${ }^{1}$ Danos acumulados nas fases de $\mathrm{R}$ e $\mathrm{M} ;{ }^{2}$ Danos acumulados nas três fases de avaliação. 

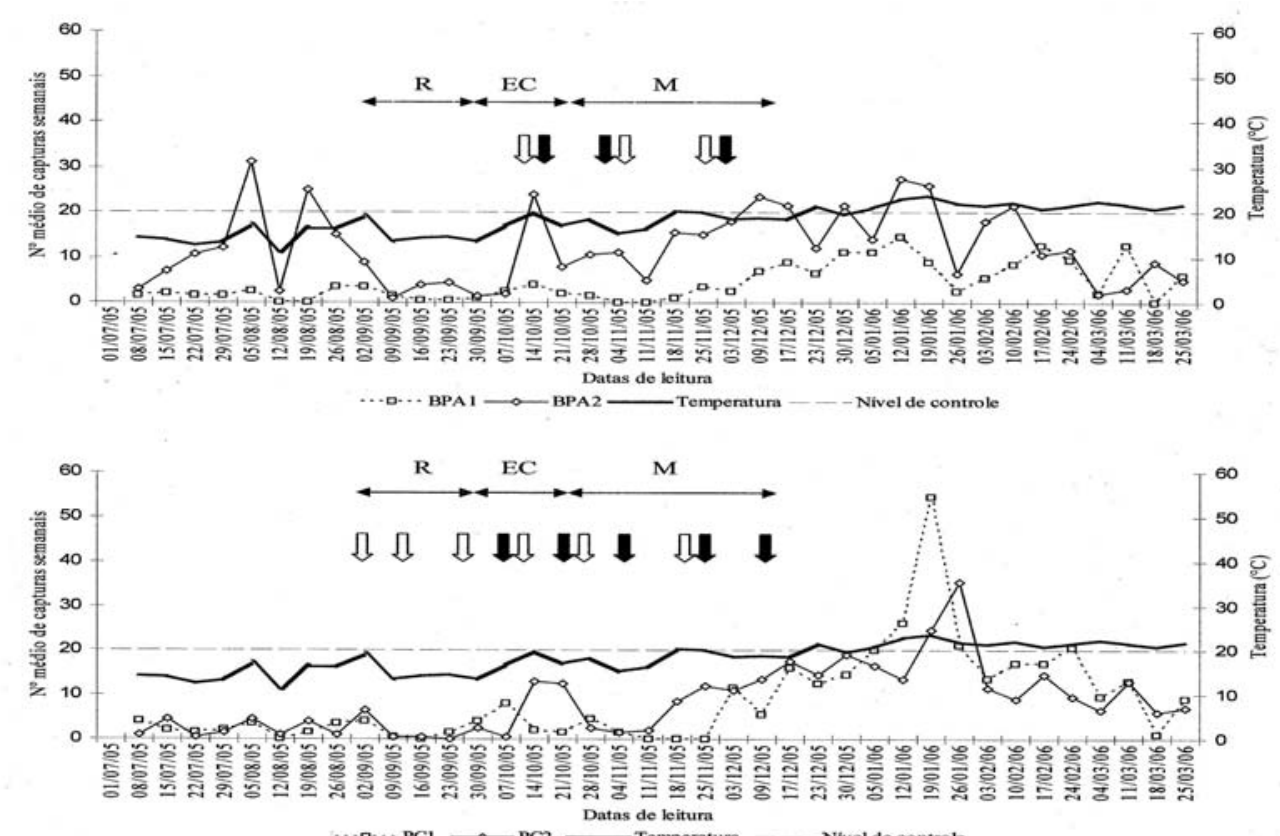

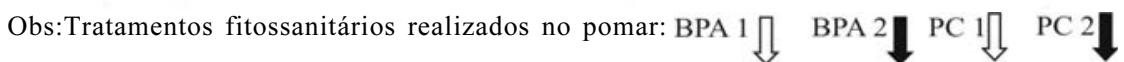

FIGURA 1 - Capturas de Grapholita molesta com feromônio (Delta), instaladas em pomares de pessegueiro 'Chimarrita' conduzidos nos sistemas de Boas Práticas Agrícolas (BPA) e produção convencional (PC), nas fases de raleio (R), endurecimento do caroço (ER) e maturação (M). Araucária-PR. Julho de 2005 a março de 2006.

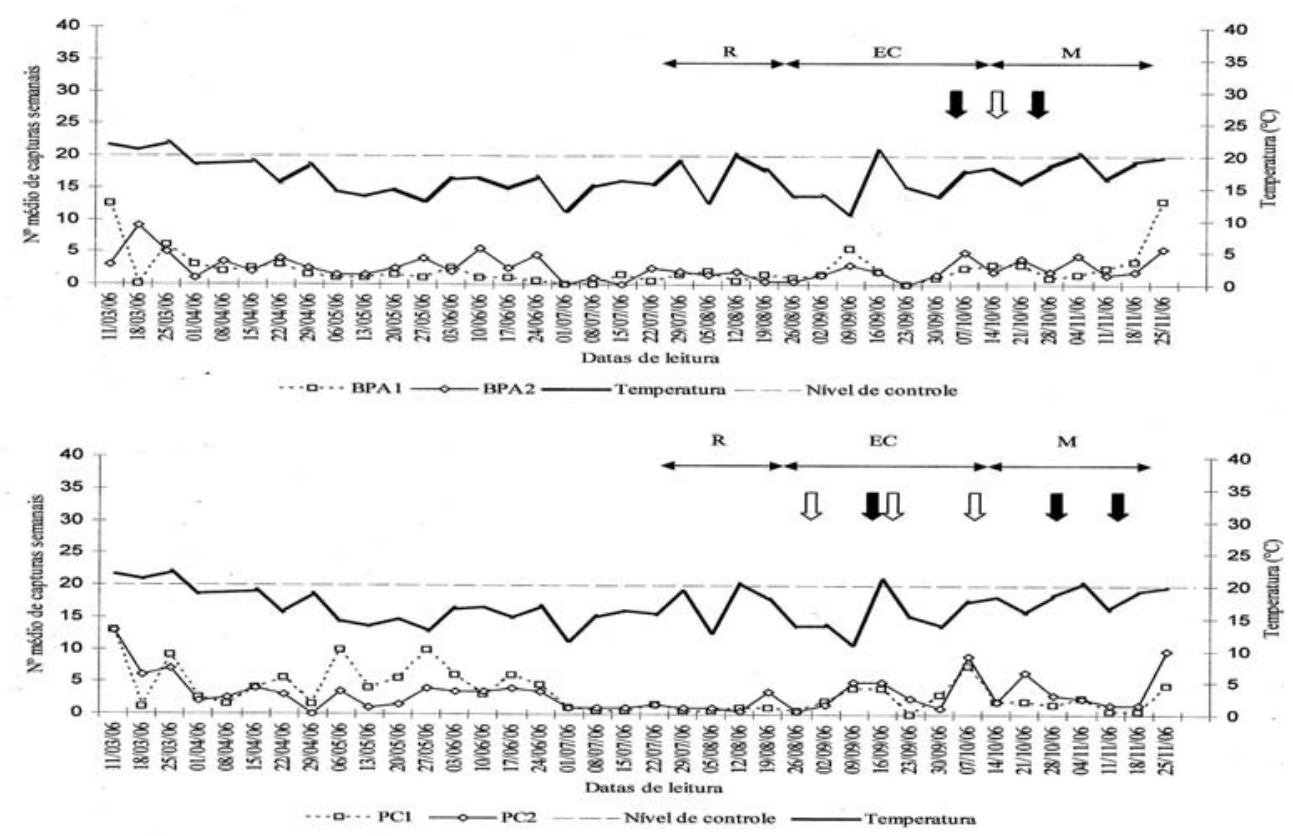

Obs:Tratamentos fitossanitários realizados no pomar:BPA 1

FIGURA 2 - Capturas de Grapholita molesta com feromônio (Delta), instaladas em pomares de pessegueiro 'Chimarrita' conduzidos nos sistemas de Boas Práticas Agrícolas (BPA) e produção convencional (PC), nas fases de raleio (R), endurecimento do caroço (ER) e maturação (M). Araucária-PR. Março a novembro de 2006.

Tratamentos fitossanitários realizados no pomar: 


\section{CONCLUSÕES}

1- As populações de G. molesta são maiores após a colheita dos frutos.

2-O controle em calendário (produção convencional) não evita danos em frutos causados por G. molesta. Não houve diferença de danos em brotações.

3- A fase maturação foi a mais suscetível da cultivar Chimarrita ao ataque de G. molesta.

\section{REFERÊNCIAS}

ANDREI, E. Compêndio de defensivos agrícolas: guia prático de produtos fitossanitários para uso agrícola. 6. ed. São Paulo: Andrei Editora, 1999.

ARIOLI, J.C.; CARVALHO, G.A.; BOTTON, M. Flutuação populacional de Grapholita molesta (Busck) com armadilhas de feromônio sexual na cultura do pessegueiro em Bento GonçalvesRS, Brasil. Ciência Rural, Santa Maria, v.32, n.1, p.1-5, 2005.

BOTTON, M.; ARIOLLI, C.J.; COLLETTA, V.D. Monitoramento da mariposa-oriental Grapholita molesta (Busck, 1916) na cultura do pessegueiro. Bento Gonçalves: Embrapa Uva e Vinho, 2001.4p. (Comunicado técnico, 30)

CARVALHO, R.P.L. Manejo integrado de pragas do pessegueiro. In: CROCOMO, W.B. (Org.). Manejo integrado de pragas. São Paulo: UNESP, 1990, p. 323-358.

CHITARRA, M.I.F.; CHITARRA, A.B. Pós-colheita de frutas e hortaliças: fisiologia e manuseio. 2. ed. Lavras: UFLA, 2005. 785 p.

CIVIDANES, F.J.; MARTINS, I.C.F.. Flutuação populacional e previsão de gerações de Grapholita molesta (Busck, 1916) (Lepidoptera: Tortricidae) em pomares de pessegueiro, Prunus persica (Linnaeus) Batsch. Acta Scientia Agronômica, Maringá, v. 28, n. 3, p. 399-405, 2006.

CRUZ, I. B. M.; TAUFER, M.; OLIVEIRA, A.K. Estudos toxicológicos. In: MALAVASI, A.; ZUCCHI, R. A. Mosca-dasfrutas de importância econômica no Brasil: conhecimento básico e aplicado. Ribeirão Preto: Editora Holos, 2000. cap. 8, p.143-150.

FACHINELLO, J.C.; COUTINHO, E. F.; MARODIN, G.; B.; BOTTON, M.; MAY DE MYO, L.L. Normas técnicas e documentos de acompanhamento da produção integrada de pêssego. Pelotas: Universidade Federal de Pelotas, 2003. 92 p.

FADINI, M. A. M.; LOUSADA, J. C. N. Impactos ambientais da agricultura convencional. Informe Agropecuário, Belo Horizonte, v.22, n.213, p.24-29, 2001

GONRING, A.H.R.; PICANÇO, M.MOURA,;M.F.; BACCI, L.; BRUCKNER, C.H. Seletividade de Inseticidas Utilizados no
Controle da Grapholita molesta (Busch) (Lepidoptera: Olethreutidae) em Pêssego, a Vespidae Predadores. Anais da Sociedade Entomológica do Brasil, Piracicaba, v.28, n.2, 1999.

GONZALEZ, H.R. Las polillas de la fruta en Chile (Lepidoptera: Tortricidae; Pyralidade). Santiago: Universidade de Chile, 2003. 188 p. (Serie Ciencias Agronômicas, 9).

GRELLMANN, E.O.; LOECK A.E.; SALLES, L.A.B.; FACHINELLO, J.C. Necessidades térmicas e estimativa do número de gerações de Grapholita molesta (Busck, 1916) (Lepidoptera: Olethreutidae) em Pelotas-RS. Pesquisa Agropecuária Brasileira, Brasília. v.27, n.7. p.999-1004, 1992.

HICKEL, E.R.; HICKEL, G.R.; DE SOUZA, O.F.F.; VILELA, E.; MIRAMONTES, O. Dinâmica populacional da mariposa oriental em pomares de pessegueiro e ameixeira. Pesquisa Agropecuária Brasileira, Brasília v. 38, n. 3, p. 325-337, 2003.

LÖECK, E.A.; BERTOLDI, L.H.M.; SALLES, L.A.B. Dinâmica populacional e estimativa do número de gerações de G. molesta (Lepidoptera: Tortricidae) na região de Pelotas-RS. Anais da Sociedade Entomológica do Brasil, Londrina, v.1, n.20, 1991.

MARTINS, G.A.K.; LINK, D. Nível de infestação da mariposaoriental em pessegueiros, resultados preliminares. Revista Centro Ciências Rurais, Santa Maria, v.8, n.3, p.263-267, 1978.

MONTEIRO, L.B.; HICKEL, E. Pragas de importância econômica em fruteiras de Caroço. In: MONTEIRO, L. B.; MAY DE MIO, L. L.; SERRAT, B. M.; MOTTA, A. C.; CUQUEL F.L. Fruteiras de Caroço: uma visão ecológica. Curitiba: UFPR, 2004. 309 p.

NUNES, J.L.S.; FARIAS R.M.; GUERRA, D.S.; GRASSELLI, V.; MARODIN, G.A.B. Flutuação populacional e controle da mariposa-oriental (Grapholita molesta Busck, 1916) em produção convencional e integrada de pessegueiro. Revista Brasileira de Fruticultura, Jaboticabal, v.25, n.2, p.227-228, 2003.

SALLES, L. A. B.; MARINI, L.H. Etiologia do ataque das lagartas de Grapholita molesta (Busck, 1916) (Lepidoptera: Tortricidae) em pessegueiros. Anais da Sociedade Entomológica do Brasil, Piracicaba, v.18, n.2, p.337-345,1989.

SALLES, L.A. Mariposa-oriental, Grapholita molesta (Lepidoptera: Tortricidae). In: VILELA, E.F.; ZUCCHI, R.A.; CANTOR, F. Histórico e impacto das pragas introduzidas no Brasil. Ribeirão Preto: Editora Holos, 2000. 173p.

SALLES, L.A.B. Grafolita (Grapholita molesta): bioecologia e controle. Pelotas: EMBRAPA-CNPFT, 1991.13p. (Documentos, 42).

SEAB - Secretaria da Agricultura e Abastecimento do Paraná. Disponível em: <http://celepar07web.pr.gov.br/agrotoxicos>. Acesso em : 30 jan. 2008. 